\title{
HIGH RESOLUTION LASER SPECTROSCOPY OF SMALL MOLECULES AND CLUSTERS
}

\author{
V. BEUTEL, H.-J. BÖHM, W. DEMTRÖDER, H.-A. ECKEL, \\ J. GRESS, M. KUHN and A. SASSO \\ Fachbereich Physik, Universität Kaiserslaŭtern 6750 Kaiserslautern, Germany
}

The combination of high resolution laser spectroscopy in collimated cold molecular beams with mass selective detection allows investigations of rotationally resolved molecular spectra of selected molecular species in the presence of molecules with overlapping absorption spectra. This is illustrated by some examples, such as the isotope selective spectroscopy of $\mathrm{Ag}_{2}$-dimers or the sub-Doppler spectroscopy of $\mathrm{Na}_{3}$ in supersonic beam with a broad mass distribution of different clusters. Time resolved laser spectroscopy of excited states of alkali molecules, perturbed by bound dark states or by repulsive states, yields information about singlet-triplet mixing and predissociation rates.

KEY WORDS: Laser, spectroscopy, molecules, clusters.

\section{INTRODUCTION}

The combination of several experimental techniques from different fields of physics and chemistry has brought about considerable progress in molecular spectroscopy and molecular dynamics for the last years. These techniques comprise:

1) Adiabatic cooling of molecules in supersonic seeded beams, resulting in a compression of the thermal population distribution into the lowest vibrationalrotational levels of the electronic ground state, which leads to a drastic simplification of otherwise complex molecular absorption spectra. ${ }^{1}$

2) A reduction of the Doppler-width of absorption lines in collimated molecular beams, when a tunable single mode laser is tuned through the absorption spectra of molecules in the beam. ${ }^{2}$

3) Two-colour two-photon ionization of molecules in combination with mass selective detection which allows a safer assignment of spectra in samples with several molecular species or mixtures of different isotopes. This technique can be realized with pulsed lasers and time of flight mass spectrometers ${ }^{3}$ or with $\mathrm{cw}$ lasers and quadrupole mass spectrometers. ${ }^{4}$

4) Time resolved spectroscopy with time resolution ranging into the picosecond or even femto-second range, which opens the possibility of investigating inter- and intra-molecular dynamics, lifetimes, fast relaxation processes ${ }^{5}$ and which even can resolve the time scale of molecular vibrations. ${ }^{6}$

The present paper illustrates these techniques by some examples, taken mainly 
from recent work in our labs in Kaiserslautern. There are, however, important activities and results also in many other labs on related problems using similar techniques. $^{7,8}$

\section{ISOTOPE SELECTIVE SPECTROSCOPY OF $\mathrm{Ag}_{2}$-DIMERS}

Although several papers have appeared on the spectroscopy of $\mathrm{Ag}_{2},{ }^{9,10}$ no rotationally resolved spectrum has been measured so far. We report here on high resolution isotope-selective rotationally resolved spectroscopy of $\mathrm{Ag}_{2}$ dimers, formed in a cold supersonic argon beam seeded with silver vapor. The measurements yield molecular constants in different electronic states, the ionization energy of $\mathrm{Ag}_{2}$ and extrapolations of the dissociation energy.

The experimental set up is shown in Figure 1. The $\mathrm{Ag}_{2}$ molecules are formed during the adiabatic expansion of an argon-silver vapor mixture $\left(p_{A_{r}} \approx 3\right.$ bar, $T \approx 2000 \mathrm{~K}$ ) from a high temperature oven through a $70 \mu \mathrm{m}$ nozzle into the vacuum. The molecular beam is collimated by a skimmer and is then crossed perpendicularly by two superimposed laser beams. The first, narrow band pulsed dye laser is tuned through the wanted electronic band system of $\mathrm{Ag}_{2}$, thus populating levels $\left(v^{\prime}, J^{\prime}\right)$ in an excited electronic state. These levels are then ionized by a second laser (either an excimer laser or another dye laser pumped by the same excimer laser).

The ions are extracted by an electric field, enter a time of flight mass spectrometer and are recorded after their mass specific flight time by a gated box car integrator.

Figure 2 shows a section of the $2 \leftarrow 0$ band in the $A^{1} \Sigma_{u} \leftarrow X^{1} \Sigma_{g}^{+}$system recorded without mass selection. One clearly recognizes the three band heads of the three isotopes ${ }^{107} \mathrm{Ag}^{107} \mathrm{Ag},{ }^{107} \mathrm{Ag}^{109} \mathrm{Ag}$ and ${ }^{109} \mathrm{Ag}^{109} \mathrm{Ag}$, which are shifted against each other. However, the $P$ - and $R$-branches of the three isotopes overlap, which makes an unambigious assignment difficult, because the "lines" in Figure 2 are in fact overlapping rotational transitions with different $J$ values of the three isotopes.

With the time of flight spectrometer the three isotopes can be separately but simultaneously recorded by three boxcar integrators with appropriate gate times (Figure 3). The calculated isotope shifts of corresponding rotational lines in the three spectra greatly facilitates the rotational assignments. The modulation of the rotational intensities, seen in Figure 3, which is due to overlapping $P$ - and $R$-lines of the same isotope, can also be utilized for a confirmation of the correct rotational assignment.

Of particular interest is the ionization potential of $\mathrm{Ag}_{2}$. It can be determined in different ways: One method is based on the direct one-photon ionization starting with thermally populated levels $\left(v^{\prime \prime}, J^{\prime \prime}\right)$ in the $X^{1} \Sigma_{g}$ ground state. Since in vapor cells or furnaces at high temperatures the thermal population distribution $N\left(v^{\prime \prime}, J^{\prime \prime}\right)$ is very broad, the ion yield, i.e. the number of produced ions as a function of the ionizing laser wavelength, follows a more or less flat curve, determined by the convolution of population densities $N\left(v^{\prime \prime}, J^{\prime \prime}\right)$ and ionization probability. In such cases, the onset of this curve, which yields the ionization potential, cannot be determined very accurately.

A more selective ionization can be achieved by resonant two-photon two-colour 


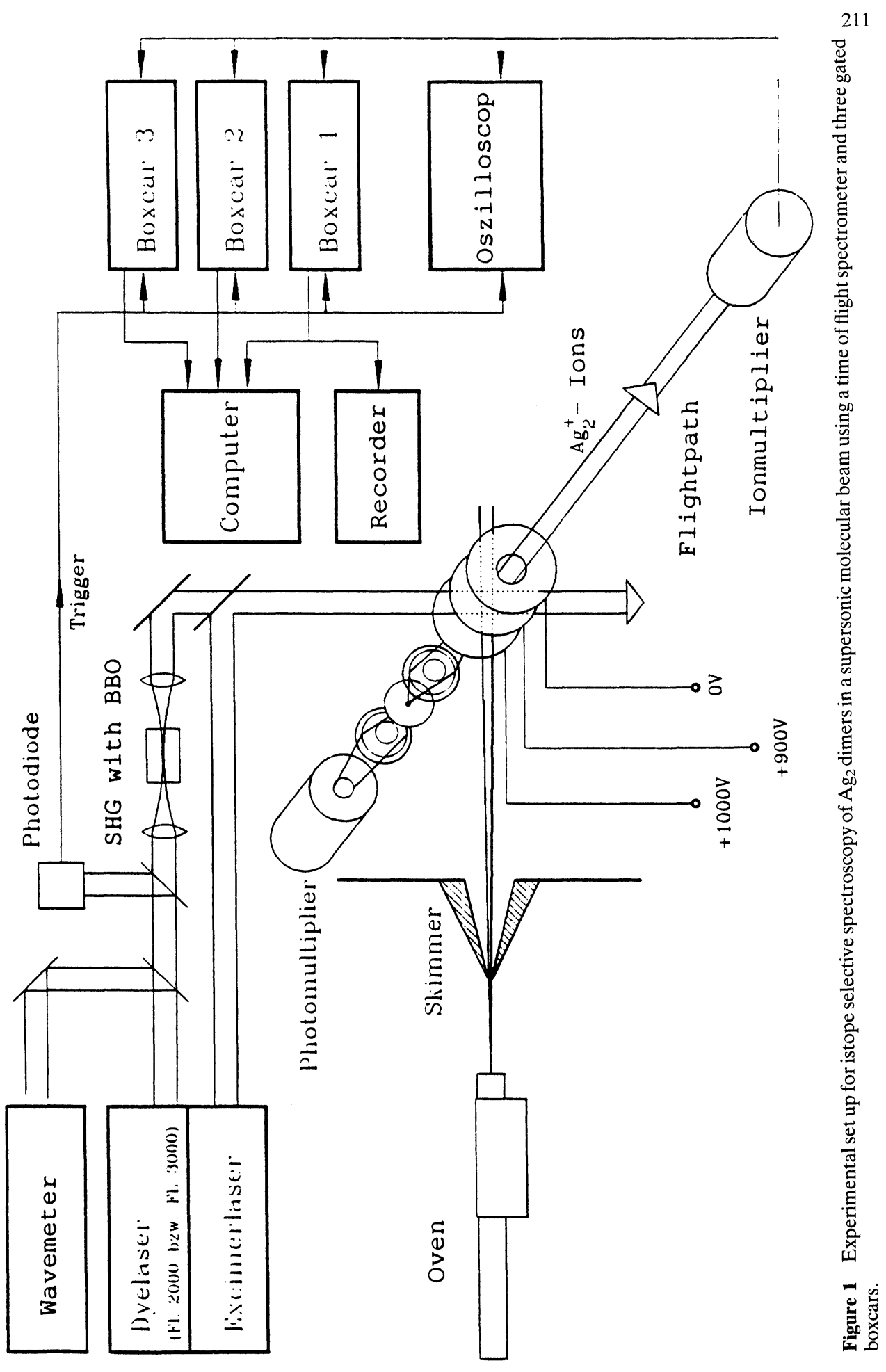


212

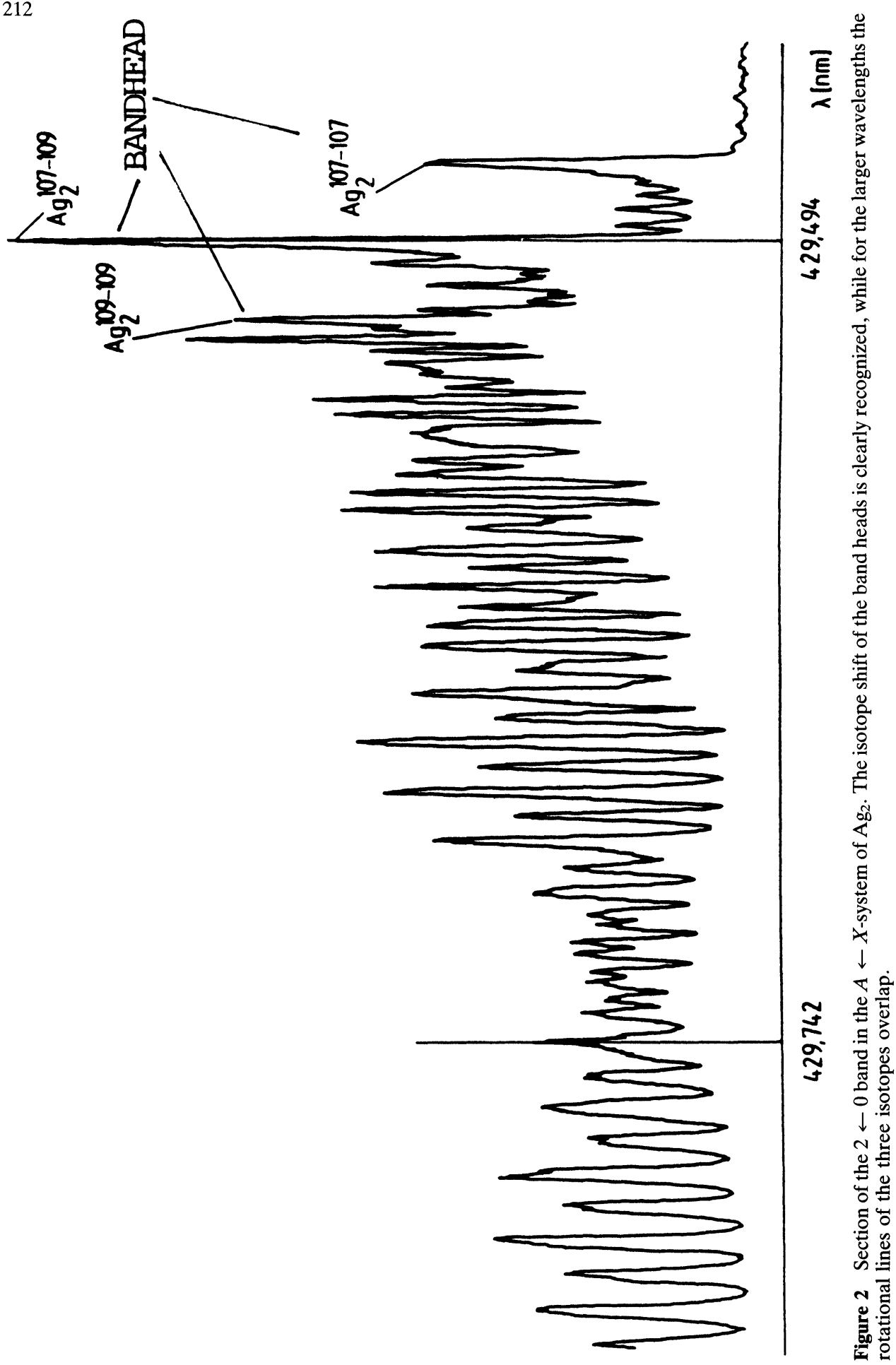



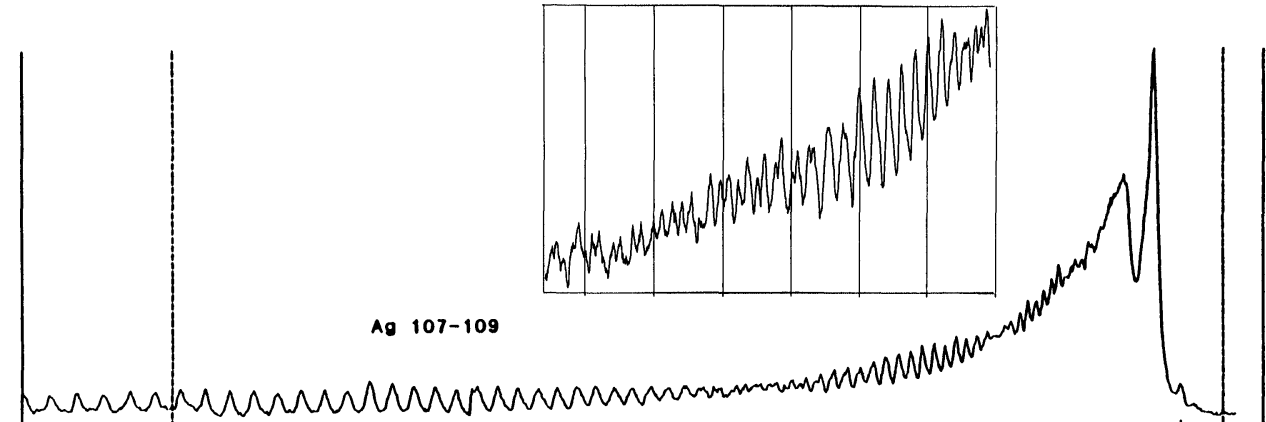

Ag 107-109

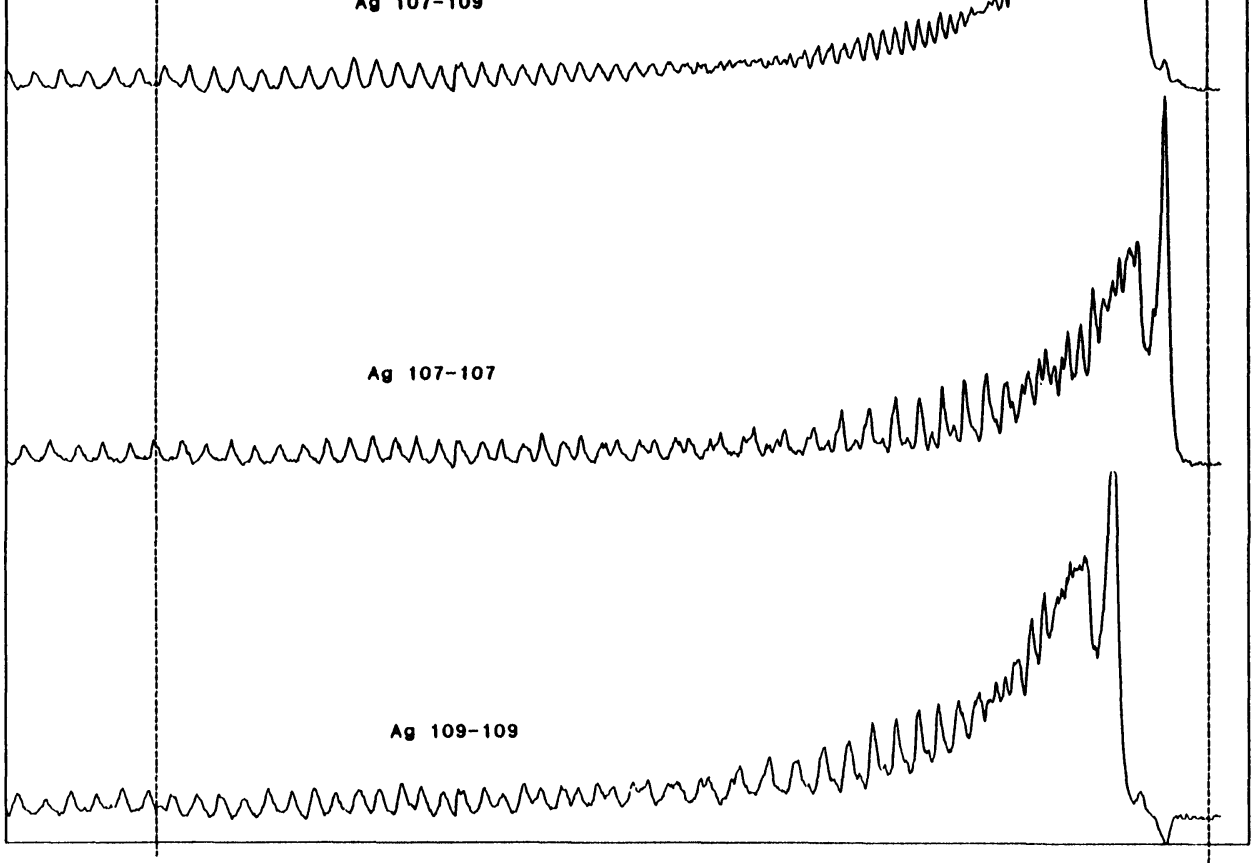

$278.2734 \mathrm{~nm}$

$278.0831 \mathrm{~nm}$

Figure 3 The $1 \leftarrow 0$ band in the $B \leftarrow X$-system of $\mathrm{Ag}_{2}$, recorded with a frequency doubled pulsed dye laser simultaneously for the three isotopes by three boxcar integrators. The insert shows an enlarged section of a spectrum.

ionization with two tunable lasers. The first laser is tuned to a selected transition $\left(v^{\prime}, J^{\prime}\right) \leftarrow\left(v^{\prime \prime}, J^{\prime \prime}\right)$ from the ground state to a level $\left(v^{\prime}, J^{\prime}\right)$ in an intermediate electronic state and a second tunable laser ionizes the excited molecules in level $\left(v^{\prime}, J^{\prime}\right)$.

The main problem of both methods is the uncertainty of a vibrational level $v^{+}$, reached in the ion ground state. If the potential curve of the ion ground state is shifted against that of the initial level of the ionizing transition, vertical transitions generally end in excited vibrational levels of the ion ground state and the apparent ionization threshold is too high. The second method has the advantage that different vibrational levels of the intermediate state can be selectively populated, thus probing 
different vertical transitions. A comparison of the measured onsets of the ionization yield curves generally allows the correct assignment of $v^{+}$and therefore the adiabatic ionization potential.

Since the ions or photoelectrons are extracted by an electric field $E$, the appearance potential AP of the ions is lowered by an amount

$$
A P=I P-\left(\frac{e^{3}}{\pi \epsilon_{0}} E\right)^{1 / 2}
$$

against the true ionizational potential $I P$. One therefore has to measure $A P$ at different electric fields and extrapolate to $E \rightarrow 0$.

The most accurate method for the determination of the ionization potential is based on the measurement of series of autoionizing Rydberg levels with term values $T\left(n, v^{*}, J^{*}\right)$ which converge for $n \rightarrow \infty$ against the level $\left(v^{+}=v^{*}, J^{+}=J^{*}\right)$ of the ion ground state. ${ }^{11}$ If several series $T\left(n, v^{*}, J^{*}\right)$ with different vibrational levels can be assigned even the vibrational and rotational constants of the molecular ion ground state can be determined. ${ }^{12}$

With the last two methods the IP of $\mathrm{Ag}_{2}$ has been determined as

$$
\operatorname{IP}\left(\mathrm{Ag}_{2}\right)=61742 \pm 4 \mathrm{~cm}^{-1}
$$

The difference between the extrapolated appearance potential and the Rydberg convergence limits is about $3 \mathrm{~cm}^{-1}$. The uncertainty of $\pm 4 \mathrm{~cm}^{-1}$ is only partly due to the wavelength measurement of the second laser (performed with a monochromator) and the uncertainty of the extrapolation from the lowest measured vibrational level $v^{*}=3$ to the level $v^{*}=0$. The main contribution comes from the unresolved rotational distribution in the band heads of the vibrational bands, which were selected (because of intensity reasons) for the two step excitation.

Isotopic specific rotationally resolved spectra, such as that shown in Figure 3, have been measured for several bands in the $B, C, D$ and $E$-electronic states of $\mathrm{Ag}_{2}$. The analysis of these spectra, which is not yet completed, yields the rotational and vibrational constants, the potential curves and the transition probabilities.

\section{SUB-DOPPLER SPECTROSCOPY OF THE $\mathrm{Na}_{3}$-CLUSTER}

Sodium metal clusters are good candidates to study the transition range between molecules, liquid drops and solid metal. They can be produced during the adiabatic expansion of a noble gas-sodium vapor mixture in a high temperature oven through a $50 \mu \mathrm{m}$ nozzle into vacuum. The $\mathrm{Na}_{3}$-system has been studied in detail by Wöste and coworkers with pulsed lasers. ${ }^{13}$

The spectral resolution of these pulsed experiments was limited by the laser bandwidth and was not sufficient to resolve the rotational structure. On the other hand, information on the geometry of $\mathrm{Na}_{3}$ is essential not only to test ab initio calculations ${ }^{14}$ but also to gain more detailed insight into the structure of a molecule without rigid geometry. The $\mathrm{Na}_{3}$-cluster represents a good example of a small 
"floppy" molecule with a shallow potential, Jahn-Teller distortion and pseudorotation. ${ }^{15}$

In order to resolve the rotational structure, sub-Doppler spectroscopy with single mode cw dye lasers is necessary. Such measurements have been performed for $\mathrm{Na}_{3}$ in our group. ${ }^{16}$ The experimental arrangement is shown in Figure 4. Cold sodium clusters $\mathrm{Na}_{\mathbf{x}}$ are formed in a supersonic argon/sodium beam during the adiabatic expansion from a heated stainless steel oven $\left(T=750-900 \mathrm{~K}, P_{A r_{r}} \leqslant 10 \mathrm{bar}\right)$. The molecular beam is skimmed and is crossed perpendicularly by the beam of a cw dye laser tuned to the $A \leftarrow X$-system of $\mathrm{Na}_{3}$. The laser induced fluorescence is viewed by a photomultiplier PM1 or can be spectrally resolved by a monochromator at a fixed excitation wavelength. In a second crossing point, located in the ion source of a quadrupole mass spectrometer collinearly to the laser beam, the dye laser excites levels in the $A$ state which are then ionized by an argon ion laser.

Efficient resonant two-photon ionization with $\mathrm{cw}$ lasers in a molecular beam requires a special experimental arrangement. The ionization step must take place before the excited level decays. With decay times of $10^{-8} \mathrm{~s}$ the molecules travel at $\bar{v}=10^{3} \mathrm{~m} / \mathrm{sec}$ a distance of $10^{-5} \mathrm{~m} \approx 10 \mu \mathrm{m}$. This implies that the two laser-beams must overlap within $10 \mu \mathrm{m}$. The experimental realization (Figure 5) uses a combination of

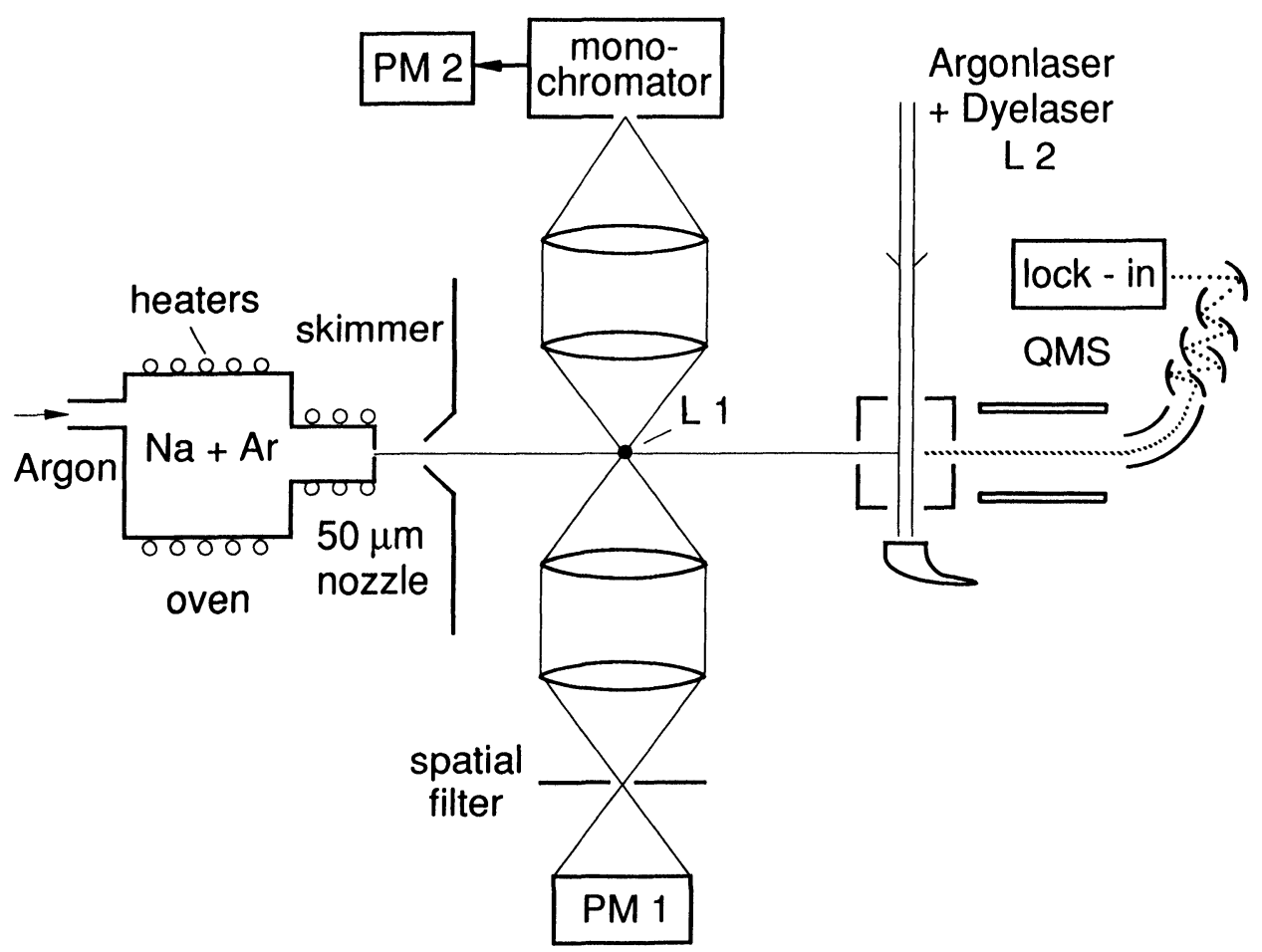

Figure 4 Experimental arrangement of sub-Doppler Spectroscopy of $\mathrm{Na}_{\mathrm{x}}$-clusters. At the first crossing point LIF can be observed, at the second crossing point in the ion course of the quadrupole mass filter resonant two-photon ionization is performed. 


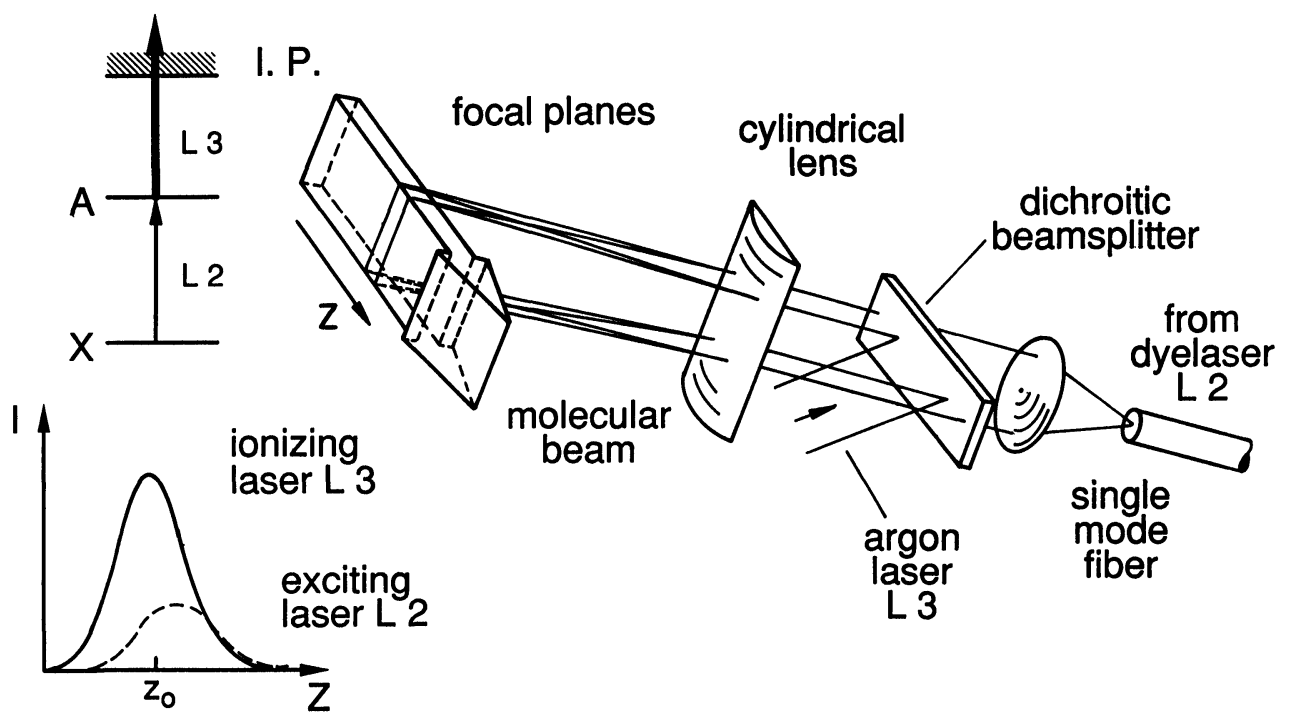

Figure 5 Efficient two-photon ionization of molecules in a molecular beam with two cw lasers.

spherical and cylindrical lenses to form a rectangular focal spot of dimensions $(20 \times 1000 \mu \mathrm{m})$ in the molecular beam so that all molecules in the beam pass through both laser beams.

A section of the sub-Doppler spectrum is shown in Figure 6. The high density of lines which, even at a resolution of $30 \mathrm{MHz}$, are only partly resolved, is due to the rotational structure of a nonrigid asymmetric top with fine- and hyperfine structure. The spectrum represents perpendicular transitions with $\Delta K= \pm 1$, a very narrow $Q$-branch (because the geometry of upper and lower state is very similar), and weaker $P$ - and $R$-branches. Although the general structure of the spectrum can be reproduced using ab initio calculations of molecular constants, ${ }^{16}$ the unambiguous assignment of individual lines turns out to be very difficult without further information.

This information is provided by optical-optical double resonance techniques (Figure 7). A dye laser L1 is tuned to a selected transition $|k\rangle \leftarrow|i\rangle$ of the $A \leftarrow X$-system, and depletes the population $N_{i}$ due to partial saturation of the transition. If $\mathrm{L} 1$ is chopped at a frequency $f_{1}$, the population $N_{i}$ is accordingly modulated. In the second crossing point the resonant two-photon ionization with a second dye laser and an argon laser takes place. When the ion signal on $M\left(\mathrm{Na}_{3}^{+}\right)$is measured after a lock-in amplifier tuned to the chopping frequency $f_{1}$, only those transitions are monitored which start from the labelled level $|i\rangle$. In order to discriminate against ionization signals caused by two argon laser photons the second dye laser L2 may be chopped at a frequency $f_{2}$ and the OODR-signal is then recorded at the sum-frequency $f_{1}+f_{2}$.

For a single lower labelled level one expects at worst three OODR-transitions with 


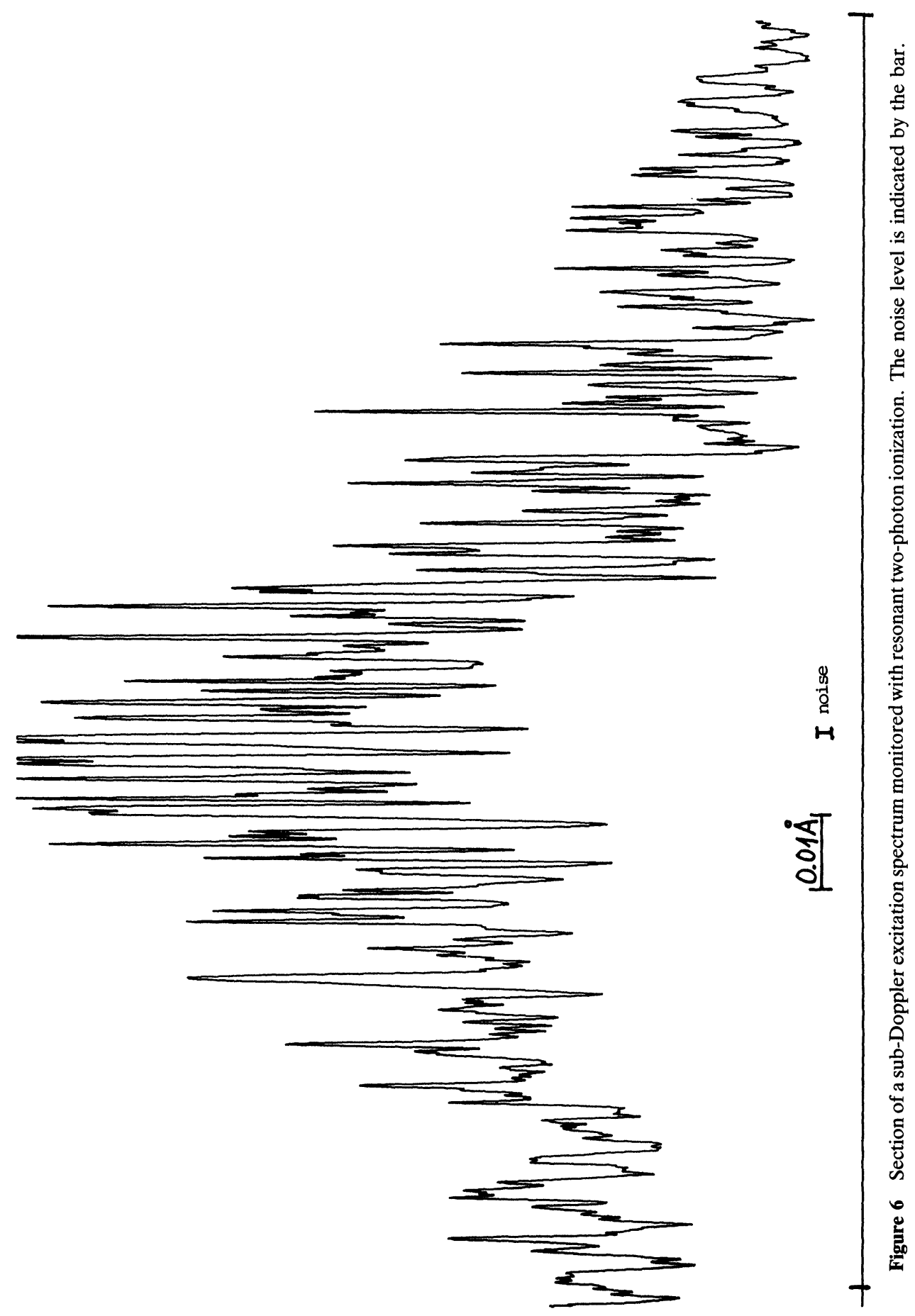




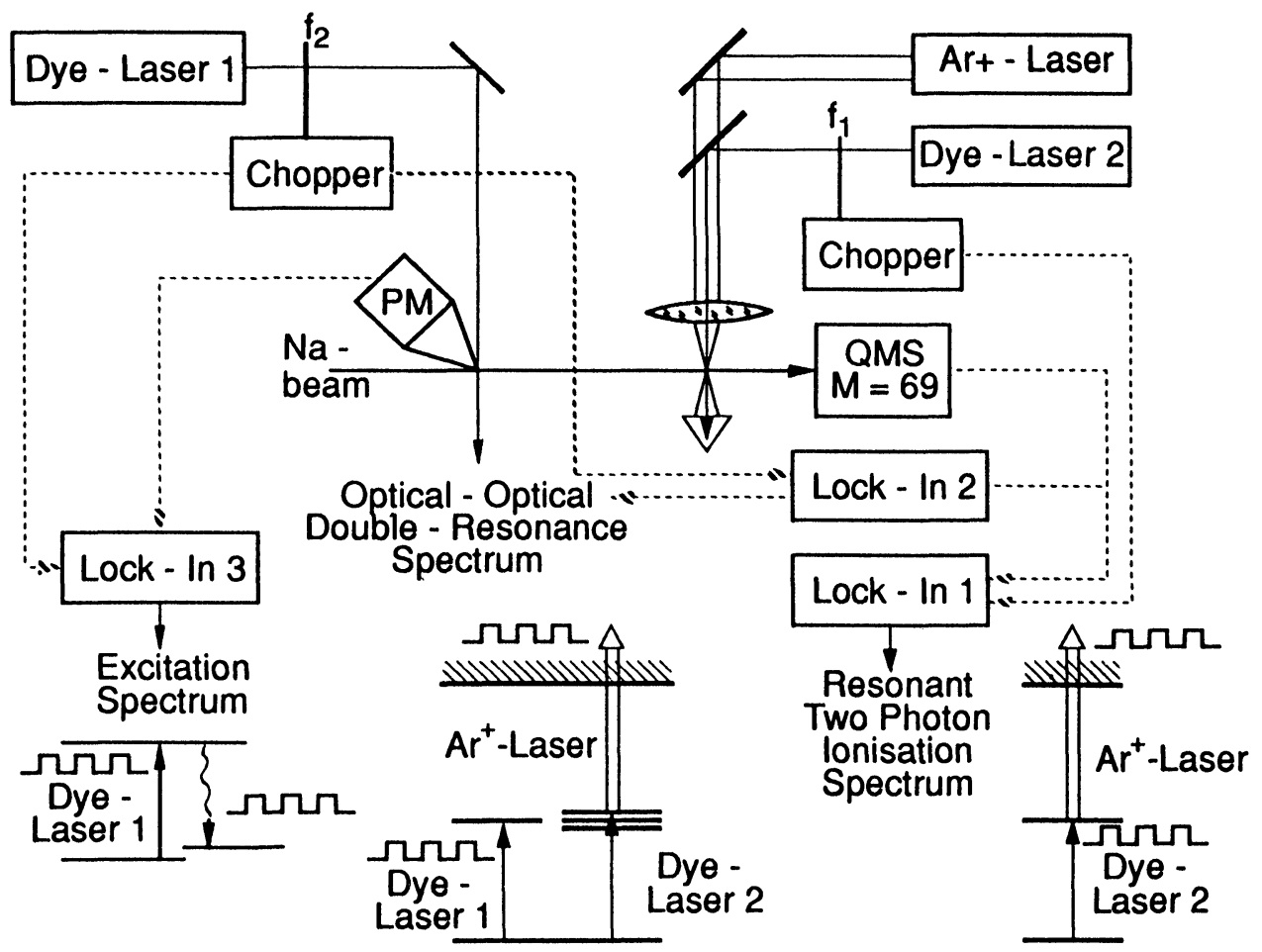

Figure 7 Arrangement of optical-optical double resonance spectroscopy where the OODR-signals are monitored by resonant two-photon ionization.

$\Delta J=0, \pm 1$ from this level into three rotational levels in the upper state. Figure 8 shows, however, that for the pump transition chosen three groups of transitions are observed which represent the hyperfine structure of the rotational transitions.

$A b$ initio calculations yield rotational constants sufficiently accurate that, at least for transitions with low rotational quantum numbers, the rotational assignments can be performed unambiguously from the measured positions and spacings of the OODR-signals. For the example of Figure 8 the ratios $[\bar{v}(R)-\bar{v}(Q)] /[\bar{v}(Q)-\bar{v}(P)]$ yield $N_{K_{a} k_{c}}^{\prime \prime}=202$. Measurements of several of such OODR signals obtained with the pump laser kept on different absorption lines in Figure 6 finally allow the assignment of the whole rotational structure of the $A \leftarrow X$-spectrum. ${ }^{17}$

When the probe laser is kept on one of the OODR-signals while the pump laser is tuned around the pump transition, the lower curve of Figure 8 is obtained. This shows the degree of saturation (about $50 \%$ ) and it also demonstrates that several different pump lines contribute to the OODR-signal. This is due to the partial overlap of lines in the dense spectrum of Figure 6.

The explanation of the hyperfine structure of the OODR-signals is more complex ${ }^{16}$ and can be traced to the slow pseudo-rotation of the $\mathrm{Na}_{3}$ molecule in the ${ }^{2} X$-ground 


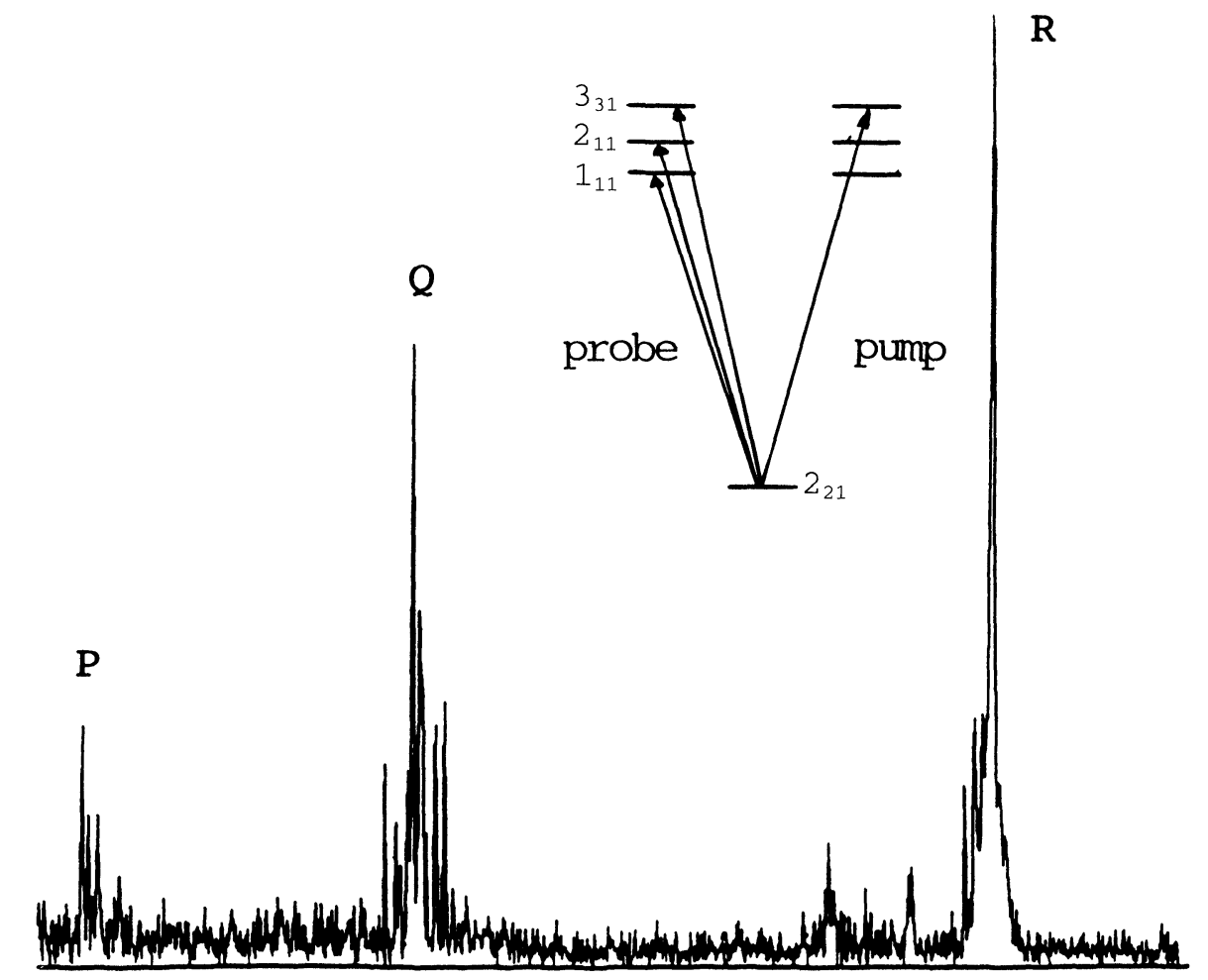

14894.714

14894.983

14895.443

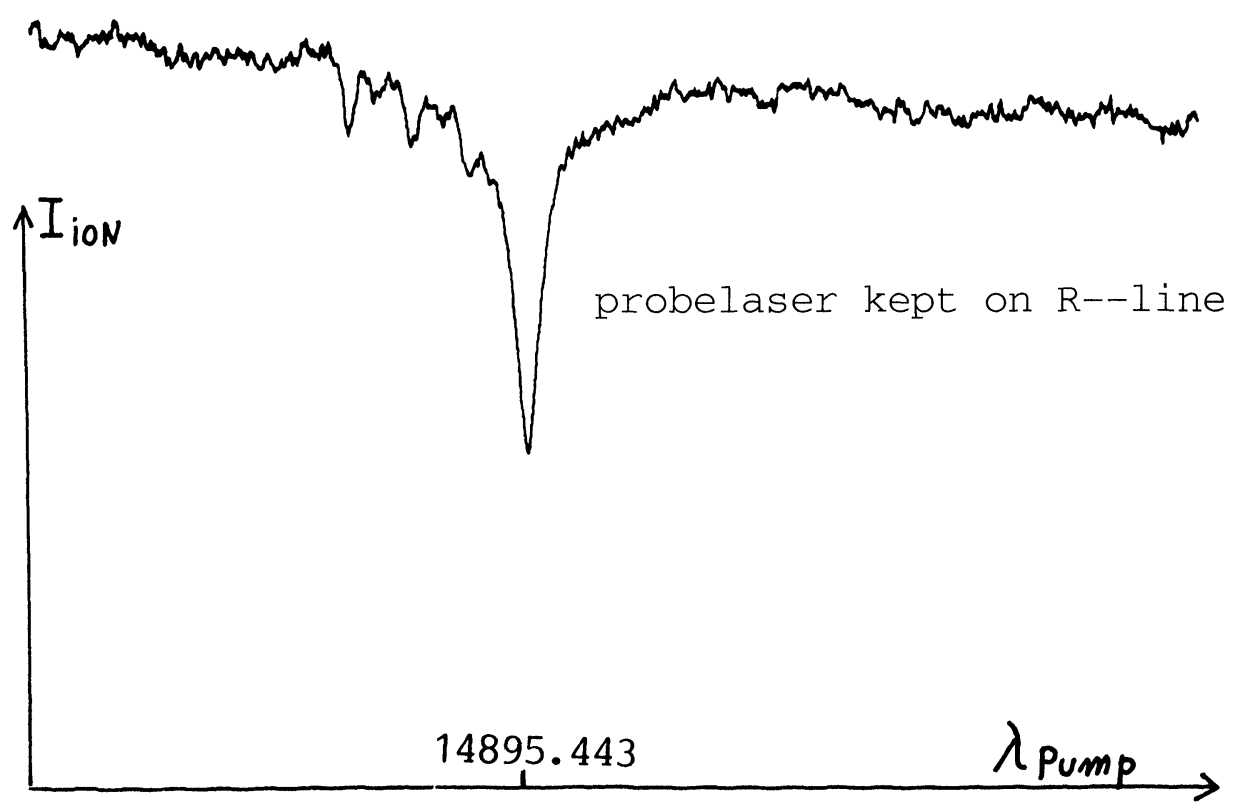

Figure $8 P, Q$ and $R$-OODR-signals obtained when the dye laser L1 is kept on the $R$-transition $3_{1 / 2} \leftarrow 2_{02}$ in the $A \leftarrow X$ system of $\mathrm{Na}_{3}$. The lower trace is obtained when the probe laser wavelength is kept constant while the pump laser L1 is tuned. It shows a depletion of the level $N_{K a K c}^{\prime \prime}=202$ by about $50 \%$. 
state, which mixes hfs components during the time between pumping and probing the molecule. Also the anomalous intensity ratios of $P, Q$ and $R$-OODR-transitions can be explained by pseudo-rotation. ${ }^{17}$

In the literature the question has been discussed whether the higher vibronic levels in the $\mathrm{Na}_{3}$ ground state may exhibit chaotic behavior. ${ }^{18}$ This may be experimentally proved by measuring the statistics of the nearest neighbour distribution of vibronic levels ${ }^{19}$ by stimulated emission pumping. Such experiments are under way in our laboratory.

\section{LIFETIMES OF PERTURBED MOLECULAR LEVELS}

Excited molecular levels may not only decay by spontaneous emission of radiation, but also by radiationless transitions, such as predissociation or collisional quenching. The total deactivation probability of a level $|i\rangle$ is then the sum of the different contributions:

$$
A_{i}^{\mathrm{total}}=A_{i}^{\mathrm{rad}}+A_{i}^{\mathrm{nr}}+N \cdot \bar{v} \cdot \sigma^{\text {quench }}
$$

Measuring the effective lifetime $\tau_{i}^{\text {eff }}=1 / A_{i}^{\text {total }}$ for different levels $|i\rangle$ as a function of the density $N$ of collision partners allows the determination of all terms in Eq. (1).

In a Stern-Vollmer plot, where $1 / \tau_{i}^{\text {eff }}$ is plotted versus $N$ one obtains from (1) a straight line with a slope $\operatorname{tg} \alpha=\bar{v} \cdot \sigma_{l}^{\text {quench }}$ and an intercept $(N=0)$ which yields the collision-free lifetime $\tau_{i}=\left(A_{i}^{\mathrm{rad}}+A_{i}^{\mathrm{nr}}\right)^{-1}$.

In the present work, alkali molecules in a heat pipe are excited by optical pulses of a synchroneously pumped mode-locked and cavity dumped tunable dye laser system. ${ }^{20}$ The pulses have a duration of $\Delta T=500 \mathrm{ps}$, a spectral band width of $\Delta v \approx 1.26 \mathrm{GHz}$ (which is nearly Fourier-limited) and a repetition rate, which can be controlled between 0 and $4 \mathrm{MHz}$.

For $\mathrm{Cs}_{2}$ molecules the average spacing of rotational lines in the spectral region $500-700 \mathrm{~nm}$ is smaller than their Doppler-width. ${ }^{21}$ The laser pulses therefore populate simultaneously several upper levels $\left(v^{\prime}, J^{\prime}\right)$ due to overlapping absorption lines. In order to measure the lifetime of a single upper level, the laser induced fluorescence has to be dispersed by a monochromator which is set to a fluorescence line emitted from the wanted upper level.

The fluorescence photons are detected by a fast photomultiplier and the decay curve is measured with the delayed coincidence single photon counting technique. ${ }^{22}$ For each excited level $|i\rangle$ a Stern-Vollmer plot is measured which yields the collisional quenching rates and the collision-free lifetimes $\tau\left(v_{i}^{\prime}, J_{i}^{\prime}\right)$.

If the upper states are perturbed, the effect of these perturbations on the lifetimes of individual rovibronic levels $\left(v_{i}^{\prime}, J_{i}^{\prime}\right)$ depends on the kind of perturbation. For heterogeneous perturbations the coupling matrix element depends on the rotational quantum number $J^{\prime}$ (rotational perturbations). ${ }^{23}$ Although for homogeneous perturbations (such as spin-orbit coupling, electrostatic interactions or vibronic coupling) the coupling matrix element itself does not depend on $J^{\prime}$, the perturbation can still depend on $J^{\prime}$ if the rotational constants of the two interaction states are different. In 
this case the energy separation $\Delta E$ between the interacting levels $\left(v_{i}^{\prime}, J_{i}^{\prime}\right)$ and $\left(v_{m}^{\prime}, J_{m}^{\prime}=J_{i}\right)$ vary with $J^{\prime}$ and the interaction is maximum for minimum $\Delta E$.

If the interaction occurs between levels $\left(v_{i}^{\prime}, J_{i}^{\prime}\right)$ of a bound state and the continuum of a repulsive state (with $\Delta E=0, \Delta J=0$ ) the predissociation rate depends on the vibrational overlap of the two wavefunctions and it has a maximum at the crossing point of the two potential curves.

In this case the collisionless lifetime $\tau_{i}$ will have a minimum. This is illustrated by Figure 9, which shows measured lifetimes of various rotational levels $J^{\prime}$ in the $D^{1} \Sigma_{u}\left(v^{\prime}=43\right)$ vibrational state of $\mathrm{Cs}_{2}$. This state is perturbed by a repulsive ${ }^{3} \Sigma_{u}$ state which dissociates into

$$
\mathrm{Cs}_{2}\left({ }^{3} \Sigma_{u}\right) \rightarrow \operatorname{Cs}\left(6^{2} P_{3 / 2}\right)+\operatorname{Cs}\left(6^{2} S_{1 / 2}\right) .
$$

The crossing points of the three fine structure components of the repulsive ${ }^{3} \Sigma_{u}$ states occur at different $J^{\prime}$ values where the lifetimes of the bound levels $\left(v^{\prime}, J^{\prime}\right)$ in the $D^{1} \Sigma_{u}$ state show pronounced minima due to predissociation.

Pumping $\mathrm{Cs}_{2}$ molecules into predissociating levels of the $E$-state results in a fast predissociation into the $5 \mathrm{D}_{3 / 2}$ state. With pulsed lasers one can achieve sufficient

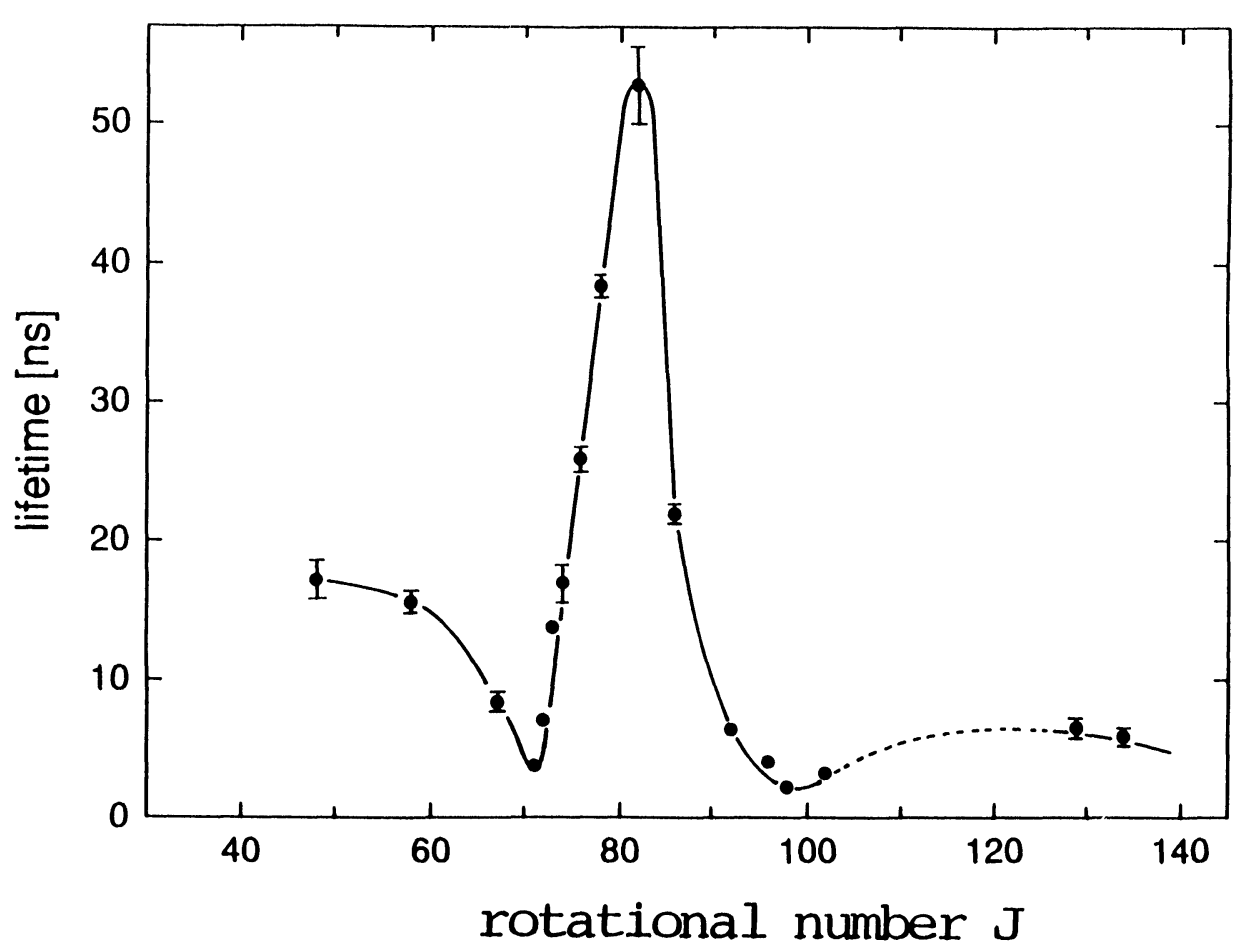

Figure 9 Lifetimes of selectively excited rotational levels in the $D^{1} \Sigma_{u}\left(v^{\prime}=43\right)$ state of $\mathrm{Cs}_{2}$ perturbed by $a^{3} \Sigma_{u}$ state with a repulsive potential curve. 
inversion to reach laser oscillation on the atomic $5 \mathrm{D}_{3 / 2} \rightarrow 6 \mathrm{P}_{1 / 2}$ transition at $\lambda=3.01 \mu \mathrm{m} .{ }^{24}$

This predissociation can be also utilized to populate the $5 \mathrm{D}_{\mathrm{J}}$ levels in order to measure the lifetimes of these atomic states which can be populated from the atomic ground state only by dipole-forbidden but quadrupole-allowed transitions. ${ }^{25}$

The $D^{1} \Sigma_{u}$ state is also perturbed by a bound ${ }^{3} \Pi_{u}$ state. This perturbation leads to a prolongation of the lifetime, if the perturbing levels have a longer lifetime than that of the perturbed levels.

From the variations of the lifetimes $\tau_{i}\left(v_{i}^{\prime}, J_{i}^{\prime}\right)$ the matrix elements of the interaction between the mutually perturbing states can be deduced. Accurate measurements of lifetimes of perturbed molecular levels therefore give detailed information about radiative transition probabilities and coupling matrix elements of radiationless transitions.

\section{CONCLUSIONS}

This paper could only give a short overview on advantages and possibilities of some methods of laser spectroscopy which are based on a combination of different experimental techniques. In particular the combination of high spectral resolution with mass selectivity allows the analysis of otherwise not accessible molecular spectra.

\section{References}

1. D. H. Levy, L. Wharton and R. E. Smalley, "Laser spectroscopy in supersonic jets" in: Chemical and Biochemical Applications of Lasers, Vol. II ed. by C. B. Moore (Academic Press, New York 1977).

2. W. Demtröder, "Laserspektroskopie" 2, Auflage (Springer, Heidelberg 1991).

3. H. J. Neusser, V. Boesl, R. Weinkauf and E. W. Schlag, "High resolution laser mass spectrometry" Int. Journal of Mass Spectr. and Ion Processes 60, 147 (1984).

4. W. Demtröder, R. Duchowicz, J. Gress, H.-J. Foth, R. Kullmer, G. Persch and M. Schwarz, Phys. Scripta T23, 176 (1988).

5. P. M. Felker, Wm. R. Lambert and A. H. Zewail, Chem. Phys. Lett. 89, 309 (1982).

6. T. Baumert, B. Bühler, M. Grosser, R. Thalweiser, V. Weiss and E. Gerber, J. Phys. Chem. in press.

7. W. Demtröder, M. Inguscio, eds. Applied Laser Spectroscopy NATO ASI Series Vol. 241 (Plenum, New York 1991).

8. S. Svanberg, "Laser spectroscopy of atoms and molecules" (Springer, Heidelberg 1990).

9. C. M. Brown and M. L. Ginter; J. Mol. Spectrosc. 69, 25 (1978).

10. D. S. Pesic and B. R. Vujisic, J. Mol. Spectrosc. 146, 516 (1991) and references therein.

11. R. F. Stebbings and F. B. Dunning, "Rydberg states of atoms and molecules" (Cambridge Press, Cambridge, MA 1983).

12. M. Schwarz, R. Duchowicz, W. Demtröder and Chr. Jungen, J. Chem. Phys. 89, 5460 (1988).

13. M. Broyer, G. Delacretaz, P. Labastie, J. P. Wolf, L. Wöste, J. Phys. Chem. 91, 2620 (1987).

14. F. Cocchini, W. Andreoni and T. H. Upton, J. Chem. Phys. 88, 6068 (1988).

15. G. Delacretaz, E. R. Grant, R. L. Whetten, L. Wöste, J. W. Zwanziger, Phys. Rev. Lett. 56, 2598 (1986).

16. H.-J. Foth, J. M. Gress, Chr. Hertzler and W. Demtröder, Z. Phys. D18, 257 (1991).

17. H.-A. Eckel, J. M. Gress and W. Demtröder, to be published.

18. J. M. Gomez Llorente, H. Taylor and E. Pollak, Phys. Rev. Lett. 62, 2096 (1989).

19. G. Persch, E. Mehdizadeh, W. Demtröder, Th. Zimmermann, H. Köppel and C. S. Cederbaum, Ber. Bunsengesellschaft Phys. Chem. 92, 312 (1988). 
20. R. H. Johnson, IEEE J. Quant. Electr. QE-9, 255 (1973).

21. M. Raab, G. Hönig, W. Demtröder and C. R. Vidal, J. Chem. Phys. 76, 4370 (1982).

22. B. Bieniak, H. P. Hinske, St. Paulus, D. Zevgolis and W. Demtröder, Ann. d. Physik 48, 15 (1991).

23. H. Levebre-Brion and R. W. Field, "Perturbations in the spectra of diatomic molecules" (Academic Press, Orlando 1986).

24. U. Diemer and W. Demtröder, Chem. Phys. Lett. 176, 135 (1991).

25. A. Sasso, W. Demtröder and J. Huennekens, Phys. Rev. submitted. 